\title{
Primary biliary cirrhosis: Environmental risk factors
}

\author{
Deepti Dronamraju, Joseph Odin and Nancy Bach* \\ Department of Liver Diseases, Mount Sinai Medical Center, New York, NY, USA
}

\begin{abstract}
Primary biliary cirrhosis (PBC) is an autoimmune disease of unclear etiology. It is a chronic, progressive condition that causes intrahepatic ductal destruction ultimately leading to symptoms of cholestasis, cirrhosis and liver failure. The disease predominantly affects middle aged Caucasian women. It has a predilection to certain regions and is found in higher incidences in North America and Northern Europe. It also has a genetic predisposition with a concordance rate of $60 \%$ among monozygotic twins. Combinations of genetic and environmental factors are proposed in the pathogenesis of this disease with a compelling body of evidence that suggests a role for both these factors. This review will elucidate data on the proposed environmental agents involved the disease's pathogenesis including xenobiotic and microbial exposure and present some of the supporting epidemiologic data.
\end{abstract}

\section{Introduction}

Primary biliary cirrhosis (PBC) is a disease of presumed autoimmune etiology that causes inflammation within the portal tracts. This leads to destruction of small and medium sized intrahepatic biliary ducts. Ultimately, this may result in symptoms of cholestasis, cirrhosis and liver failure [1]. The serologic hallmark of the disease is the production of anti- mitochondrial antibodies (AMA), known to react most frequently with the E2 subunit of private dehydrogenase complex among other autoantigens [2]. The disease predominates among women with a 10:1 female to male ratio and is usually diagnosed in middle aged women though it has been diagnosed in patients as young as 15 and as old as $93[1,3]$.

The pathogenesis of the disease remains enigmatic since the cause is likely complex and multi-factorial. The current view is that PBC occurs in a genetically susceptible population with external triggers precipitating its development. Considerable evidence points to environmental triggers involved in the development of this disease. In this review, we will focus on observed

${ }^{*}$ Corresponding author: Nancy Bach, MD, Division of Liver Diseases/ Transplant Institute, Mount Sinai Medical Center, Box 1101, 5 East 98th Street, New York, New York 10029, USA. Tel.: +1 212 241 0034; E-mail: nancy.bach@mountsinai.org. and circumstantial data pertaining to possible environmental exposures including xenobiotics, and microbes.

\section{Genetic predisposition}

The prevalence of PBC amongst families with an affected member is estimated to be 1000 times greater than the general population [4,5]. PBC has been weakly associated with the HLA-DRB $1 * 0801$ haplotype and a recent study by Hirschfield et al suggests a significant association between HLA class II, IL12A and IL12RB2 loci as well [6]. This is especially interesting as these haplotypes are associated with IL12 immunoregulatory signaling. These findings further affirm the genetic predisposition to this disease.

In 2004, Selmi et al identified 8 pairs of monozygotic twins and 8 pairs of dizygotic twins from a large cohort of patients with PBC. $60 \%$ of the monozygotic twins had PBC affecting both siblings while none of the dizygotic twins were dually affected. This concordance rate for the monozygotic twins is one of the highest of all diseases. This study confirms the strong role genetics plays in the pathogenesis of this disease [4]. However, genetics alone are not sufficient to explain the pathogenesis as 3 of the 8 monozygotic twins were not concordant for the disease. There is likely a second trigger. 
Table 1

Selected studies showing geo-clustering of primary biliary cirrhosis $[8,9,11-18]$

\begin{tabular}{lccll}
\hline $\begin{array}{l}\text { Author(s), } \\
\text { Year }\end{array}$ & $\begin{array}{c}\text { Geographic } \\
\text { clustering }\end{array}$ & $\begin{array}{c}\text { Number of } \\
\text { cases }\end{array}$ & $\begin{array}{l}\text { Site of } \\
\text { study }\end{array}$ & $\begin{array}{l}\text { Possible environmental } \\
\text { risk factor }\end{array}$ \\
\hline Triger, 1980 & Yes & 34 & Sheffield, U.K. & Water \\
Hamlyn,1983 & No & 117 & Northeast England & Sunlight \\
Triger, 1984 & Yes & 552 & Western Europe & None \\
Borda, 1989 & Yes & 50 & Navarra, Spain & None \\
Danielsson, 1990 & Yes & 111 & Northern Sweden & None \\
Myszor, 1990 & No & 347 & Northeast England & None \\
Witt-Sullivan, 1990 [16] & No & 225 & Ontario, Canada & None \\
Metcalf, 1997 & Yes & 160 & Newcastle upon Tyne, England & Urban area \\
Tsuji, 1999 & Yes & 156 & Hiroshima, Japan & Nuclear bomb \\
Prince, 2001 & Yes & 770 & Northeast England & Urban area \\
Ala, 2006 & Yes & 211 & New York City & Toxic waste site \\
\hline
\end{tabular}

Table 2

\section{Disease triggers}

PBC demonstrates a large geographic variation in its incidence and prevalence. The incidence of the disease is significantly higher in Northern Europe and North America when compared to Mediterranean and African countries [1]. In addition, disease clustering with and without environmental associations has been reported by several groups (Table 1). Abu- Mouch et al described 4 unique clusters including: a family of $10 \mathrm{sib}-$ lings with half having PBC, a husband and wife with PBC, a family of two genetically unrelated individuals with PBC, and a cluster of PBC in Alaska [7]. Their data adds to the considerable body of clinical and molecular data suggesting clustering of this disease is associated both with genetic and likely environmental triggers. The epidemiologic and molecular data presented below help support this hypothesis.

\section{Epidemiology}

The first real suggestion of disease clustering was reported by Triger in 1980 when he found clustering of PBC cases in Sheffield, England. He proposed groundwater contamination as a possible source for this clustering. However, analysis of the suspected water reservoir, showed no significant differences from the other reservoirs in the region [8]. James et al. also proposed water contamination as a possible mechanism of the PBC clustering that his group noted in urban areas of Northeast England [9].

In 2006, we investigated whether our perception of a higher prevalence of PBC from an area known to be developed on landfill sites was accurate. Superfund sites (SFS) are hazardous waste sites designated for cleanup by the Department of Environment and Conservation (DEC). We looked at prevalence rates of PBC near
Median std prevalence ratio of PBC significantly higher in clusters with SFS

\begin{tabular}{cccc}
\hline & CLUSTERS & CLUSTERS & P value \\
& WITHOUT & WITH & \\
& SFS & SFS & \\
\hline PBC & 0.51 & 0.94 & 0.001 \\
PSC & 0.28 & 0.28 & 0.572 \\
\hline
\end{tabular}

p: significance values for Mann-Whitney $U$ test, 2-tailed Std prev ratio: observed/std expected prevalence.

designated SFS in New York City. Data were collected from PBC and PSC patients in the Mount Sinai School of Medicine PBC database (MSSM PBC database). To avoid referral bias, we also collected data from all PBC and PSC patients listed for liver transplantation by the Organ Procurement Transfer Network (OPTN) in the five boroughs of New York City from 1995-2003. In the first part of our study we examined zip codes for all PBC $(n=99)$ and PSC ( $n=73)$ patients listed for liver transplant in New York City. Expected prevalence ratios, adjusted for age, gender and race were calculated. Median standardized prevalence ratios in zip codes containing/adjacent to a SFS $(n=89)$ were compared to the zip codes without SFS $(n=85)$. Median prevalence ratios for PBC were significantly higher in clusters associated with SFS compared with PSC (Table 2).

In the second part of our study, we applied SaTScan technology (a validated statistical analysis software program looking for disease clusters) to detect specific clusters of PBC near SFS using our OPTN and MSSM PBC databases [10]. Again, an increased prevalence of PBC patients was identified in clusters around SFS. Furthermore, the clusters of PBC in our cohort (MSSM PBC database) correlated with the OPTN data [11]. The major contaminants in these superfund sites were halogenated aromatic hydrocarbons including polychlorethylene and benzene. 
Since then many studies continue to show geographic clustering of PBC [8,9,11-18]. McNally et al showed space-time clustering in a population from Northeast England [19]. This phenomenon is interesting as it supports the role for a transient environmental agent in the disease pathogenesis. Space-time clustering is an epidemiologic term used to describe a large number of cases presenting in a small geographic area within a limited time period. When space time clustering is identified, a transient, possibly environmental agent becomes suspect. Using population based data from the northeast regions of England over a 16 year period (1987-2003), space time clustering was noted for PBC. The Knox test and K-function methods used in statistical determination of spatiotemporal patterns, were used in this study to analyze space-time clustering. Variations in population density were adjusted based on nearest neighbor thresholds. Individual space-time clusters were identified using Kulldorff's scan statistic. Using these methods, 1015 cases of highly significant spacetime clustering were identified $(\mathrm{P}<0.001)$. Cases diagnosed within 1-4 months of each other showed the highest degree of clustering. McNally's identification of space-time clustering using rigorous statistical tools in a stable population without migration is highly suggestive of transient environmental agent involvement in disease pathogenesis.

In the largest epidemiologic study involving PBC, Gershwin et al interviewed 1032 individuals with $\mathrm{PBC}$ and a randomly picked control group [20]. Using multivariate analysis, they were able to show multiple risk factors for developing PBC. These included: having a first degree relative with $\mathrm{PBC}$, recurrent urinary tract infections, past smoking history, use of hormone replacement therapy, and the frequent use of nail polish. Of note, an increased frequency of urinary tract infections has previously been reported in PBC [21]. As will be described in the next section, this association may be one related to molecular mimicry.

\section{Molecular data}

\subsection{Xenobiotics}

Xenobiotics are small molecular weight foreign chemicals that can complex with the body's own proteins to alter structure and induce an immunogenic response. These small compounds are found ubiquitously in our environment in household detergents, cleaners, food preservatives, pesticides and pollutants among other things. They have been implicated in the pathogenesis of other autoimmune disease. For example, systemic lupus erythematosis can be induced by the drug hydralazine and scleroderma has been linked to silica exposure [22]. In a similar manner, the involvement of xenobiotics in the pathogenesis of PBC has also been studied. Most xenobiotics are processed by the liver.

The liver is the major detoxification organ of the body and the biliary tree is thereby exposed to high levels of xenobiotics during their clearance. Gershwin et al have extensively studied the immunoenicity of modified forms of PDC-E2, the major autoantigen recognized by anti-mitochondrial antibody. They propose loss of tolerance to PDC-E2, secondary to modification of the autoantigen by xenobiotic conjugation, as a mechanism for triggering autoantibody production (AMA) in PBC [23]. Several studies have implicated halogenated compounds as the mimotope that triggers this cascade of events [24].

In one study, rabbits immunized with 6-bromohexonate, a halogenated xenobiotic complex, all developed AMA that reacted with PDC-E2 and inhibited its enzymatic function [25]. Similar results were seen in guinea pig models [26]. Interestingly, in rabbit models, tolerance was recovered and a gradual reduction in autoantibody production was documented following repeated immunization with the xenobiotic [27]. Other examples of possible xenobiotics include halothane. Halothane is an antiquated anesthetic which oxidizes to a reactive intermediate that binds to cellular proteins leading to tri fluoro acetyls (TFAs). These TFAs not only cause an immunogenic response but also cross react with $\mathrm{PDC}-\mathrm{E} 2$ [28].

2-octynoic acid has also been identified as having possible in vivo activity in modifying PDC-E2. This xenobiotic is widely used in perfumes, makeup and nail polishes possibly suggesting a linkage for the female predominance of the disease [29]. Additionally as mentioned earlier, an epidemiologic study suggested nail polish use as a risk factor for PBC. Despite the many identified immunogenic xenobiotics, identifying potential causative agents is not an easy task as exposures and molecular modifications likely take place long before a diagnosis of PBC is made. The triggering agent may no longer be present at the time of disease manifestation.

\subsection{Microbial exposure}

A number of microbes have been implicated in the pathogenesis of PBC. DNA of gram positive bacteria 
and LTA, an antigenic bacterial cell wall component, have been detected in the bile of PBC patients. It is postulated that the peri- portal inflammation seen in PBC patients may cause increased ductal permeability. This may lead to leakage of bacterial antigens into the ductal system thereby provoking an immunogenic response [30]. The suggested mechanism of action for microbial exposure causing an immunogenic response is molecular mimicry. This refers to the hypothesis that antigens on bacteria and viruses may be similar enough to host antigens to elicit an autoimmune response by host $\mathrm{T}$ cells.

Escherichia coli have been suggested as a possible culprit. This is interesting because epidemiologic studies have shown an increased incidence of urinary tract infections in the PBC population [20,21,31]. As E. coli is the most common cause for these infections; this theory is an attractive one. The PDC-E2 auto-antigen is a highly preserved protein sequence in phylogeny and is found in many prokaryotes. Shimoda et al had shown that a peptide derived from E. coli was able to activate a host response against PDC-E2. These observations further suggest the possibility of molecular mimicry in the initiation of this disease [31]. Those that refute the theory have suggested that titers of antibodies against E. coli are often much less than titers against human complex. They are also often not seen in patients with early $\mathrm{PBC}$ but rather in those with advanced disease.

Another gram negative bacterium that has been implicated in PBC is the aerobic Novosphingobium aromaticivorans. This microbe is found in soil and water and is non-pathogenic in humans. Studies have shown a high degree of homology between the sequence of human PDC-E2 and lipoylated proteins for N. aromaticivorans. The reactivity against PDC-E2 is also 100 to 1000 fold greater than that seen with E. coli antigens. One study showed $100 \%$ reactivity with the bacterium in PBC patients compared to none in the control group [32]. Another study demonstrated that autoreactive AMA and T-cell mediated autoimmunity can be induced in the biliary ducts after immunization with this bacterium [33]. However, this was done in murine models. Interestingly, the bacteria also have a role in activating estrogens suggesting a role in the female predominance of PBC [34]. Furthermore, this bacterium is commonly found in soil and water, again linking with the aforementioned epidemiologic studies of PBC.

A role for viruses in the pathogenesis of $\mathrm{PBC}$ has also been explored. One group of investigators was able to isolate human beta retrovirus in the peri-hepatic lymph nodes of PBC patients. The retrovirus bore similari-
Table 3

Proposed antigens in pathogenesis of PBC

\begin{tabular}{ll}
\hline Xenobiotics: & Benzene \\
& Polychlorethylene \\
& 6-bromohexonate \\
& 2-octynoic acid \\
& Halothane \\
& Other halogenated hydrocarbons \\
Microbes: & E. coli \\
& N. Aromaticivorans \\
& Human $\beta$ retrovirus \\
& Chlamydia \\
& P. acnes \\
& Lactobacillus \\
& Mycobacterium \\
\hline
\end{tabular}

ties to a murine mammary tumor and human retrovirus cloned from breast cancer tissue [35]. However this study could not be replicated by other groups. There have also been studies of Propionibacterium, Chlamydia and a case report of lactobacillus as causative factors of PBC; these findings have yet to be reproduced [3638]. Identifying a single causative microbe in the pathogenesis of PBC is unlikely given the natural course of the disease. It is also probable that there are multiple agents capable of inciting the immune mediated response (Table 3 ).

\subsection{Current perspective and future direction}

Rather than solving the enigma of PBC, we have presented many of the individual pieces of this still unsolved puzzle. Much of our knowledge is based on observational and circumstantial evidence. While some of the pieces seem to form a cohesive theory others may be a "red herring". The current thought remains that the pathogenesis of $\mathrm{PBC}$ is both complex and multi-factorial in nature.

Like other autoimmune conditions, the two hit hypothesis has been suggested in which an already genetically susceptible individual faces a secondary insult triggering the cascade of autoimmune events. However, neither the genetic inheritance pattern nor the inciting triggers are yet clear. Though numerous studies have supported a role for environmental agents such as xenobiotics and microbes, further work is needed to elucidate this disease's development. The development of animal models will likely help us move towards putting the pieces of the puzzle together. Clearly, great strides remain to be made in the study of $\mathrm{PBC}$ and other autoimmune diseases. 


\section{References}

[1] M.M. Kaplan and M.E. Gershwin, Primary Biliary Cirrhosis, N Engl J Med 353(12) (22 September 2005), 1261-1273.

[2] M. Gershwin, I. Mackay, A. Sturgess and R. Coppel, Identification and specificity of a cDNA encoding the $70 \mathrm{kd} \mathrm{mi-}$ tochondrial antigen recognized in primary biliary cirrhosis, $J$ Immunol 138(10) (15 May 1987), 3525-3531.

[3] Y. Dahlan, L. Smith, D. Simmonds et al., Pediatric-onset primary biliary cirrhosis, Gastroenterology 125(5) (2003), 14761479.

[4] C. Selmi, M.J. Mayo, N. Bach et al., Primary biliary cirrhosis in monozygotic and dizygotic twins: Genetics, epigenetics, and environment, Gastroenterology 127(2) (2004), 485-492.

[5] N. Bach and F. Schaffner, Familial primary biliary cirrhosis, $J$ Hepatol 20(4) (1994).

[6] G.M. Hirschfield, X. Liu, C. Xu et al., Primary Biliary Cirrhosis Associated with HLA, IL12A, and IL12RB2 Variants, N Engl J Med 360(24) (11 June 2009), 2544-2555.

[7] S.C. Abu-Mouch, G.D. Benson, T.P. Kenny, P. Invernizzi, M. Zuin, M. Podda,L. Rossaro and M.E. Gershwin, Geographic clusters of primary biliary cirrhosis, Clin Dev Immunol 10(24) (Jun-Dec 2003), 127-131.

[8] D. Triger, Primary biliary cirrhosis: an epidemiological study, Br Med J 281(6243) (20 Sep 1980), 4.

[9] J. Metcalf, R. Bhopal, J. Gray, D. Howel and O. James, Incidence and prevalence of primary biliary cirrhosis in the city of Newcastle upon Tyne, England Int J Epidemiol 26(4) (1 August 1997), 830-836.

[10] M. Kuldorff, SaTScan: software for the spatial and space-time scan statistics, Version 4.02004;Rockville, MD: Information Management Services, Inc.

[11] A. Ala, C.M. Stanca, M. Bu-Ghanim et al., Increased prevalence of primary biliary cirrhosis near superfund toxic waste sites, Hepatology 43(3) (2006), 525-531.

[12] B.P. Triger DR and J. Rodes, Epidemiology of primary biliary cirrhosis, Liver, 4(3) (Jun 1984), 195-200.

[13] H.M. Borda F, J.M. Zozaya, C. Jimenez, F.J. del Cazo, A. Lanas, G. Tiberio and M. Munoz, Primary biliary cirrhosis in Navarra, Annals of Medicine International 6(2) (Feb 1989), 63-66.

[14] A.B.L. Danielsson and P. Uddenfeldt, Epidemiology of primary biliary cirrhosis in a defined rural population in the northern part of Sweden, Hepatology 11(3) (Mar 1990), 458-464.

[15] M.J.O. Myszor, The epidemiology of primary biliary cirrhosis in northeast England: an increasingly commmon disease? Quarterly Journal of Medicine 75(276) (Apr 1990), 377-385.

[16] H.J. Witt-Sullivan, K. Cauch, L. Blendis, C. Ghent, A. Katz, R. Milner, S.C. Pappas, J. Rankin and I.R. Wanless, The demography of primary biliary cirrhosis in Ontario, Canada, Hepatology 12(1) (Jul 1990), 98-105.

[17] K. Tsuji, Y. Watanabe, J. Van De Water et al., Familial Primary Biliary Cirrhosis in Hiroshima, Journal of Autoimmunity 13(1) (1999), 171-178

[18] M.I.C.A. Prince, P. Diggle, M. Jarner, J.V. Metcalf and O.F. James, The geographical distribution of primary biliary cirrhosis in a well-define cohort, Hepatology 34(6) (Dec 2001), 1083-1088.

[19] R.J.Q. McNally, S. Ducker and O.F.W. James, Are transient environmental agents involved in the cause of primary biliary cirrhosis? Evidence from space-time clustering analysis, Hepatology 50(4) (2009), 1169-1174.

[20] M.E. Gershwin, C. Selmi, H.J. Worman et al., Risk factors and comorbidities in primary biliary cirrhosis: A controlled interview-based study of 1032 patients, Hepatology 42(5) (2005), 1194-1202.

[21] P.H.-M.J. Butler, H. Baum and A.K. Burroughs, Detection of M2 antibodies in patients with recurrent urinary tract infection using an ELISA and purified PBC specific antigens. Evidence for a molecular mimicry mechanism in the pathogenesis of primary biliary cirrhosis? BIochem Mol Biol Int 35(3) (Mar 1995), 473-485.

[22] H. Kita, X.S. He and M.E. Gershwin, Autoimmunity and environmental factors in the pathogenesis of primary biliary cirrhosis, Annals of Medicine 36(1) (2004), 72-80.

[23] M.E. Gershwin and I.R. Mackay, The causes of primary biliary cirrhosis: Convenient and inconvenient truths, Hepatology 47(2) (2008), 737-745.

[24] S.A. Long, C. Quan, J. Van de Water et al., Immunoreactivity of Organic Mimeotopes of the E2 Component of Pyruvate Dehydrogenase: Connecting Xenobiotics with Primary Biliary Cirrhosis, J Immunol 167(5) (September 1) (2001), 2956-2963.

[25] P.S.C. Leung, C. Quan, O. Park et al., Immunization with a Xenobiotic 6-Bromohexanoate Bovine Serum Albumin Conjugate Induces Antimitochondrial Antibodies, J Immunol 170(10) (15 May 2003), 5326-5332.

[26] P.S.C. Leung, O. Park, K. Tsuneyama et al., Induction of Primary Biliary Cirrhosis in Guinea Pigs following Chemical Xenobiotic Immunization, J Immunol 179(4) (15 August 2007), 2651-2657.

[27] K. Amano, P.S.C. Leung, Q. Xu et al., Xenobiotic-Induced Loss of Tolerance in Rabbits to the Mitochondrial Autoantigen of Primary Biliary Cirrhosis Is Reversible, J Immunol 172(10) (15 May 2004), 6444-6452.

[28] C. Selmi and M.E. Gershwin, The role of environmental factors in primary biliary cirrhosis, Trends in Immunology 30(8) (2009), 415-420.

[29] K. Amano, P.S.C. Leung, R. Rieger et al., Chemical Xenobiotics and Mitochondrial Autoantigens in Primary Biliary Cirrhosis: Identification of Antibodies against a Common Environmental, Cosmetic, and Food Additive, 2-Octynoic Acid, $J$ Immunol 174(9) (1 May 2005), 5874-5883.

[30] K. Tsuneyama, K. Harada, N. Kono et al., Scavenger cells with Gram-positive bacterial lipoteichoic acid infiltrate around the damaged interlobular bile ducts of primary biliary cirrhosis, Journal of Hepatology 35(2) (2001), 156-163.

[31] S. Shimoda, M. Nakamura, H. Shigematsu et al., Mimicry peptides of human PDC-E2 163-176 peptide, the immunodominant T-cell epitope of primary biliary cirrhosis, Hepatology 31(6) (2000), 1212-1216.

[32] K.A. Padgett, C. Selmi, T.P. Kenny et al., Phylogenetic and immunological definition of four lipoylated proteins from Novosphingobium aromaticivorans, implications for primary biliary cirrhosis, Journal of AutoimMunity 24(3) (2005), 209219.

[33] J. Mattner, P.B. Savage, P. Leung et al., Liver Autoimmunity Triggered by Microbial Activation of Natural Killer T Cells, Cell Host \& Microbe 3(5) (2008), 304-315.

[34] K. Fujii, S. Kikuchi, M. Satomi, N. Ushio-Sata and N. Morita, Degradation of 17 beta\}-Estradiol by a Gram-Negative Bacterium Isolated from Activated Sludge in a Sewage Treatment Plant in Tokyo, Japan. Appl. Environ, Microbiol 68(4) (1 April 2002 ), 2057-2060.

[35] L. Xu, Z. Shen, L. Guo et al., Does a betaretrovirus infection trigger primary biliary cirrhosis? Proceedings of the National Academy of SciEnces of the United States of America 100(14) (8 July 2003), 8454-8459. 
[36] K. Harada, K. Tsuneyama, Y. Sudo, S. Masuda and Y. Nakanuma, Molecular identification of bacterial 16S ribosomal RNA gene in liver tissue of primary biliary cirrhosis: Is $<\mathrm{I}>$ Propionibacterium acnes $</ \mathrm{I}>$ involved in granuloma formation? Hepatology 33(3) (2001), 530-536.

[37] D. Bogdanos, T. Pusl, C. Rust, D. Vergani and U. Beuers, Primary biliary cirrhosis following lactobacillus vaccination for recurrent vaginitis, Journal of Hepatology 49(3) (2008), 466-473.

[38] A.S. Abdulkarim, L.M. Petrovic, W.R. Kim, P. Angulo, R.V. Lloyd and K.D. Lindor, Primary biliary cirrhosis: an infectious disease caused by Chlamydia pneumoniae? Journal of Hepatology 40(3) (2004), 380-384. 


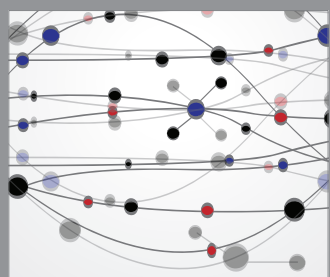

The Scientific World Journal
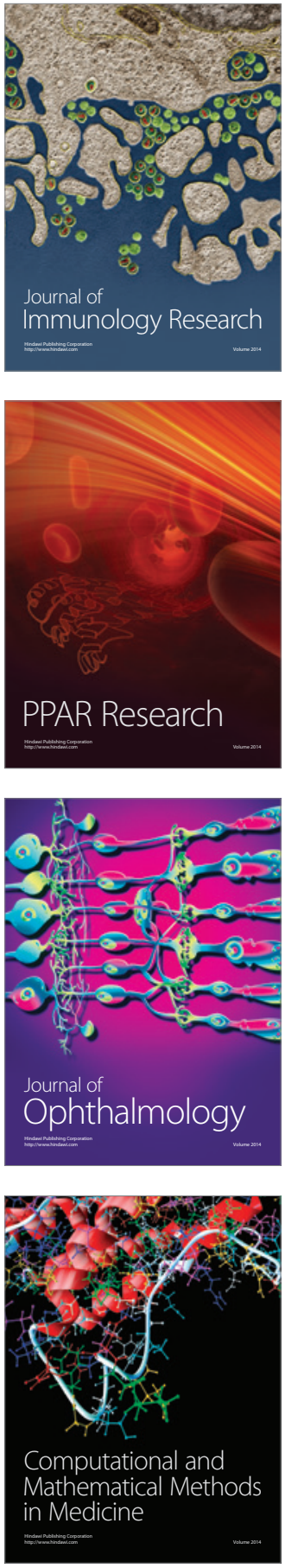

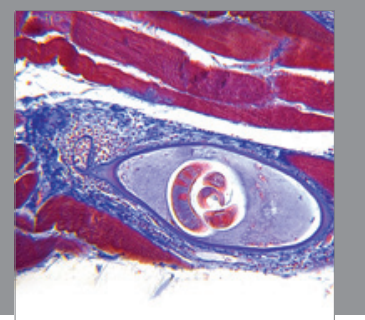

Gastroenterology

Research and Practice
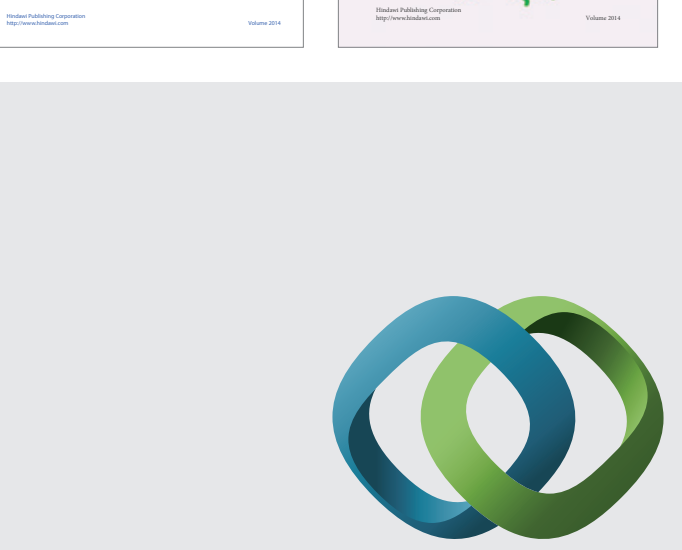

\section{Hindawi}

Submit your manuscripts at

http://www.hindawi.com
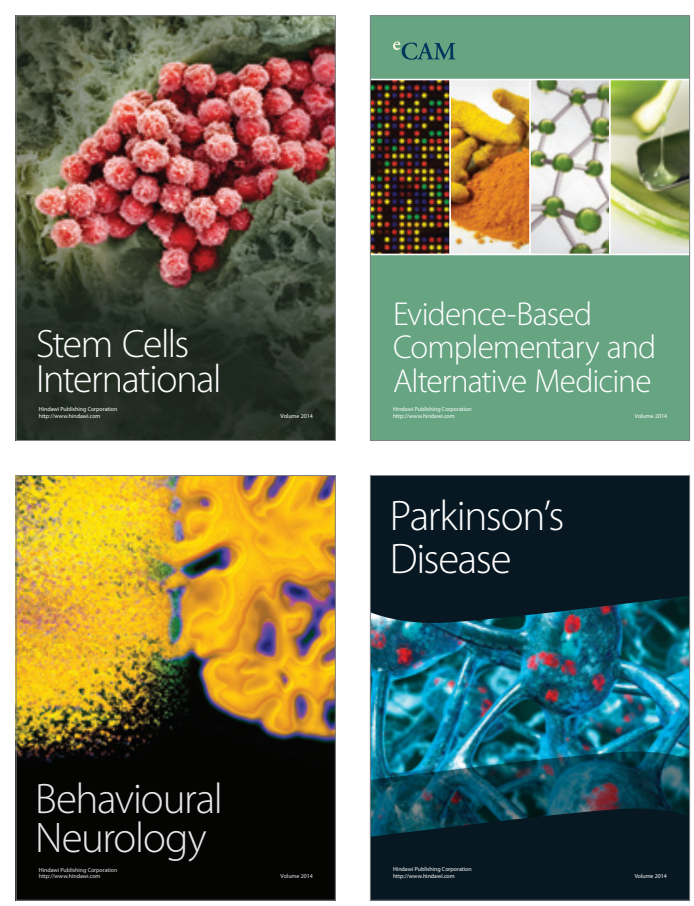

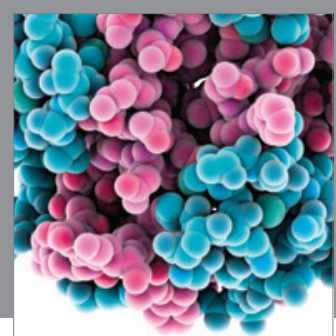

Journal of
Diabetes Research

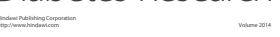

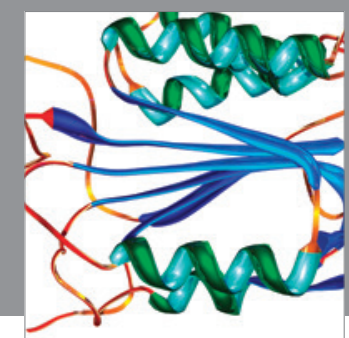

Disease Markers
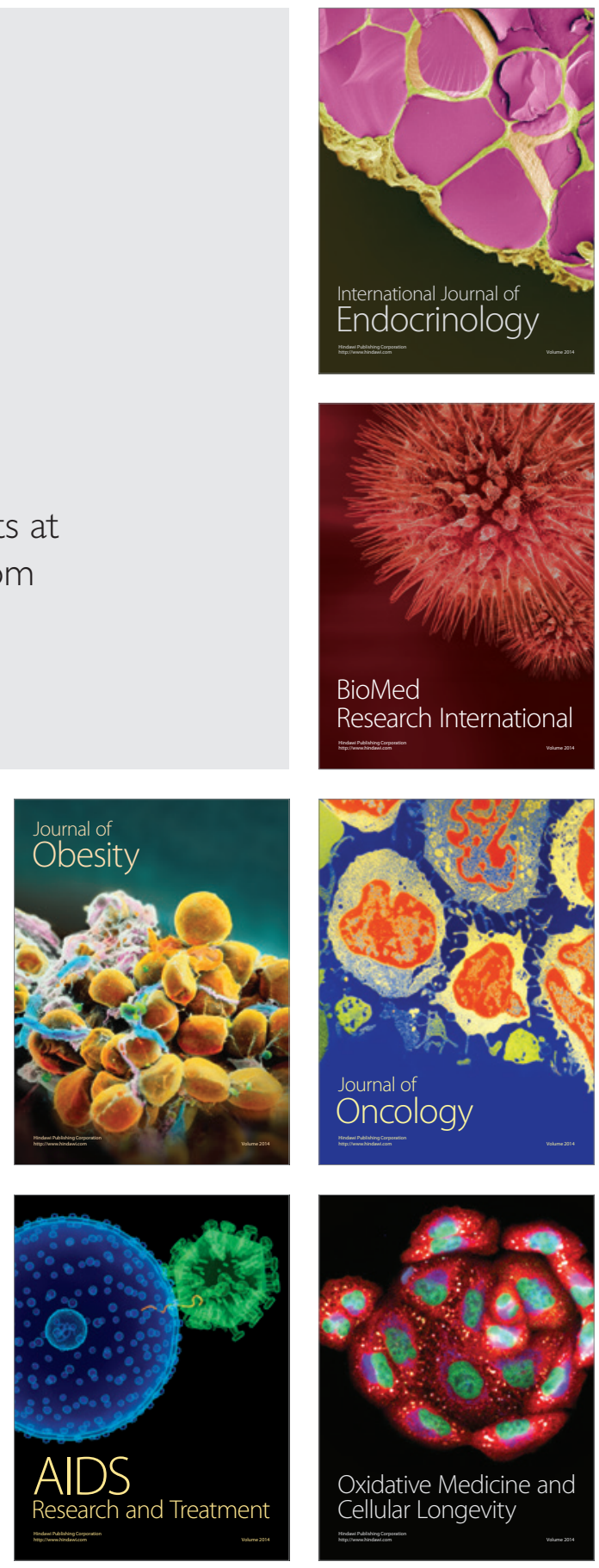